\title{
VHF ANTENNA INTEGRATED INTO A CLASSICAL FIBRE METAL LAMINATE FUSELAGE PANEL
}

\section{EMUS 2020}

\author{
J. LANSINK ROTGERINK ${ }^{*}$, C. HEUTS ${ }^{\dagger}$, J. VERPOORTE $^{*}$ AND C. VAN HENGEL ${ }^{\ddagger}$ \\ * Royal Netherlands Aerospace Centre (NLR) \\ Voorsterweg 31, 8316 PR Marknesse, The Netherlands \\ e-mail: Jesper.Lansink.Rotgerink@nlr.nl, http://www.nlr.nl \\ $\dagger$ GKN Fokker Elmo \\ Aviolandalaan 33, 4631 RP Hoogerheide, The Netherlands \\ email: Charly.Heuts@fokker.com, http://www.gknaerospace.com \\ $₫$ GKN Fokker Aerostructures \\ Industrieweg 4, 3351 LB Papendrecht, The Netherlands \\ Email: cees.vanhengel@fokker.com, web page: http://www.gknaerospace.com
}

Key words: Integrated slot antenna, VHF, parallel plate resonator, antenna miniaturization, bookleafing

\begin{abstract}
Replacement of protruding antennas by integrated alternatives will reduce the energy consumption and $\mathrm{NO}_{\mathrm{x}}$ and $\mathrm{CO}_{2}$ emissions of an aircraft. This paper proposes and integrated VHF cavity backed slot antenna that is suitable to replace blade antennas. To reduce the size of the cavity a parallel plate resonator is used. The slot is integrated into a fibre metal laminate fuselage panel. A bookleafing principle is used to reinforce the panel at the position of the slot, making sure the material quality is not reduced. Measurement results of a demonstrator antenna show the feasibility of the proposed antenna. Moreover, the paper discusses several electromagnetic and structural design trade-offs for this integrated antenna.
\end{abstract}

\section{INTRODUCTION}

Challenges set by the European Union to reach Flightpath 2050 climate goals require innovative solutions to reduce the energy consumption of aircraft. One of the focusses of the ACASIAS project is the replacement of protruding antennas on an aircraft by integrated alternatives. Specifically, this paper discusses replacement of a conventional blade antenna used for very high frequency (VHF) communications by slot antennas that are integrated in fibre metal laminate (FML) panels. Such integration of antennas in fuselage panels will reduce the aerodynamic drag and consequently the energy consumption of an aircraft.

The intention is to integrate a slot antenna into an FML fuselage panel. However, radiation of this slot antenna into the interior of the aircraft has to be prevented. Conventionally this is resolved by placing a cavity with a depth of a quarter wavelength behind the antenna. At the frequency band of the targeted blade antenna, centred around $125 \mathrm{MHz}$, this would imply an integrated antenna with a thickness up to $60 \mathrm{~cm}$, which is unacceptable. Therefore, cavity miniaturization techniques are required to minimize the thickness of the antenna without 
degrading the antenna performances. High-permittivity dielectric materials can be used, but these also generally have high mass densities. In [1]-[3] high impedance surfaces (HIS) are used to minimize the thickness of the antenna. The disadvantage of such a technique is the required space parallel to the fuselage to place the amount of HIS elements for the HIS to work properly [4]. At VHF frequencies this implies a HIS width that would not fit in between the stringers of an aircraft. Therefore, in this paper a parallel plate resonator (PPR) technique is used [5]. In a PPR the required quarter wavelength distance from the slot to a reflection plane is transformed into a folded waveguide structure, thereby reducing the thickness of the antenna. In contrast to the dual-resonator box in [5], in this paper a single-resonator PPR is used to further decrease the weight of the antenna. Several design trade-offs of such a PPR slot antenna are discussed, which include the use of dielectric materials to reduce the dimensions (but might increase weight), as well as bandwidth (BW) versus gain trade-offs.

Apart from these electromagnetic (EM) challenges the integration of a slot antenna into an FML panel raises clear structural challenges. Basically, this integration implies the creation of a hole in the skin of an aircraft, by which the strength of the skin is reduced. On top of that, the corners of the slot antennas can cause build-up of stresses. Therefore, the skin needs to be locally reinforced. In this paper, a so-called bookleafing method is proposed to make the transition from a conventional FML layup to a thicker piece of pure glass fibre below the slot antenna, back to conventional FML.

Section 2 discusses the EM design of the antenna, while section 3 covers the structural challenges caused by the integration. Section 4 discusses some important design trade-offs Section 5 gives results for a demonstrator antenna and eonclusions are given in section 6. 2 ELECTROMAGNETIC The proposed VHF antenn plate resonator and the feed stiucture. All three are discussed in this section.

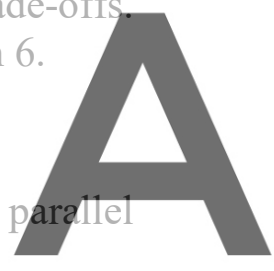

Register fon freet at thtups//www.scipedia.com to download the version without the watermark

One of the main advantages of a blade antenna, a monopole type antenna, is its nearly omnidirectional radiation. A slot antenna is the dual of a dipole antenna: a rectangular hole in a metallic ground plane. It radiates efficiently if its length is half a wavelength. Its feed induces currents in the ground plane that are enforced to flow around the slot. This causes radiation in a pattern that is again nearly omnidirectional, with only a null in the longitudinal direction of the slot. This makes a slot antenna very suitable for replacing the blade antenna.

As mentioned, the optimal length of the slot antenna is roughly half a wavelength. This implies that changing the surrounding medium of the slot will also change the optimal length of the slot. When the entire surrounding space is replaced by material of relative permittivity $\varepsilon_{r}$ then the length of the slot is reduced by a factor equal to the square root of that permittivity. When part of the surrounding medium is different from air, the optimal length can be found through simulations (in our case the full-wave software Feko was used). The resonance frequency of the slot can be found by simulation with an ideal source at the position where the real part of the slot impedance is maximal and the imaginary part crosses zero. Once the optimal slot length has been found, the PPR is added and its dimensions optimized. 


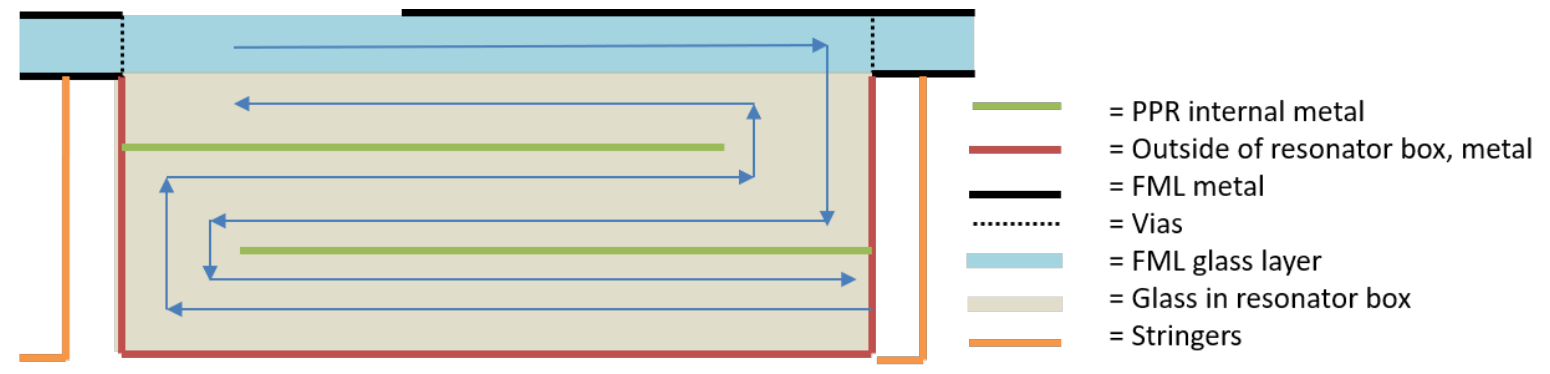

Figure 1: Illustration of a cross-section of a slot antenna integrated in FML, with PPR attached. The FML is in this case illustrated without detailed layer build-up. This will follow in section 3.

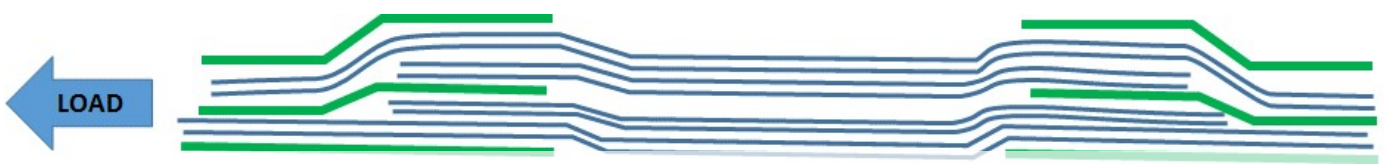

Figure 2 Transition from FML to «full glass» GFRP to FML (concept)

\subsection{Parallel plate resonator}

To prevent radiation of the slot antenna into the aircraft interior, a cavity will need to be attached to the interior side of the slot antenna. To reduce the size of such a cavity a parallel plate resonator is used, of which the concept is explained in detail in [5]. In general, the PPR is a structure in which the required distance of a quarter wavelength from slot to short-circuit is covered in a folded waveguide (see Figure
EM waves entering the PPR travel half a wave
these waves leave the slot in phase with the way
fuselage. Thus, the length of the PPR channel inside the PPR, which is the guided wavelength:

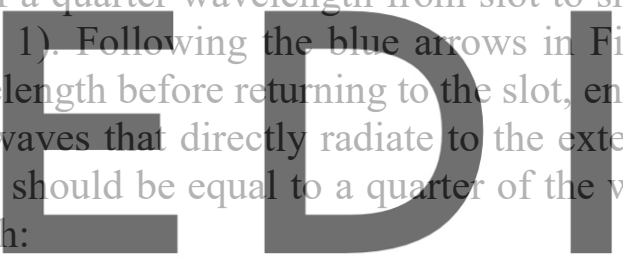

1

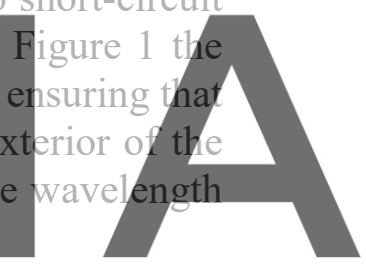

(1)

Register for free at https//www.scipedia.com to d ${ }_{1}$ ownload the version without the watermark

Here $c$ is the wave velocity in the medium of the PPR, $f$ is the frequency and $w$ is the length of the PPR in the dimension orthogonal to the cross-section shown in Figure 1.

The design of a PPR depends on a large number of parameters, such as the total length of the channel, the height of the channel, material properties, the length of the plates internal to the PPR box and even the dimensions of the slot. In general, the recipe is to first design the proper slot dimensions, after which the PPR dimensions can also be tuned by using an ideal source. Once the slot and PPR are tuned such that the resonance is at the correct frequency, the feed structure should be added to tune the overall antenna behavior.

\subsection{Antenna feed}

Feeding the slot antenna is done by a microstrip feed that extends over the slot. For optimal matching the correct feed position should be found. For a slot antenna, the impedance is high in the centre of the slot and zero at the end of the slot. The optimal feed position is the position at which the real part of the slot impedance matches the impedance of the microstrip feed. As in [5], here a $50 \Omega$ feed and a $100 \Omega$ stub is chosen. The stub is the part of the feed 
that extends behind the slot. By tuning the length of the stub the imaginary part of the input impedance of the slot will be counteracted. The antenna and feed are well matched if the reflection coefficient of the source is as low as possible, but at least lower than $-6 \mathrm{~dB}$. The higher the frequency band over which this condition holds, the higher the bandwidth of the antenna. This bandwidth can be improved by optimizing the feed and stub to introduce a second resonance [6]. By placing this resonance close to the original slot resonance the bandwidth can be enhanced. As a final solution to optimize the bandwidth of the antenna a matching network can be used to improve the matching of the antenna. This matching network is formed by lumped inductances and capacitances and is used to get rid of slight mismatches between the antenna and source impedances.

\section{STRUCTURAL REINFORCEMENTS BY BOOKLEAFING PRINCIPLE}

The need to create a slot in the metallic layers to act as antenna causes a significant issue for the structural performance of the basic skin material, because it removes about $64 \%$ of the load carrying material. The skin is a Glare3-3/2-0.3 material, meaning that it consists of 3 metal layers (interspersed with 2 composite layers: hence 3/2) of $0.3 \mathrm{~mm}$ thickness. In Glare 3, each composite layer consists of one 0 and one 90 degrees-oriented glass fibre prepreg ply of $0.127 \mathrm{~mm}$ thickness each. Thus, at the slot, the removal of $3 \times 0.3 \mathrm{~mm}$ removes $0.9 \mathrm{~mm}$ of the total thickness of $1.4 \mathrm{~mm}$. Such a material "thinning" would result in an unacceptable stress increase degrading the structural performance. To restore the stress level to acceptable levels, the existing ones. In used in composite struct

a "bookleafing" principl Secondly, the "repai" autoclave curing and
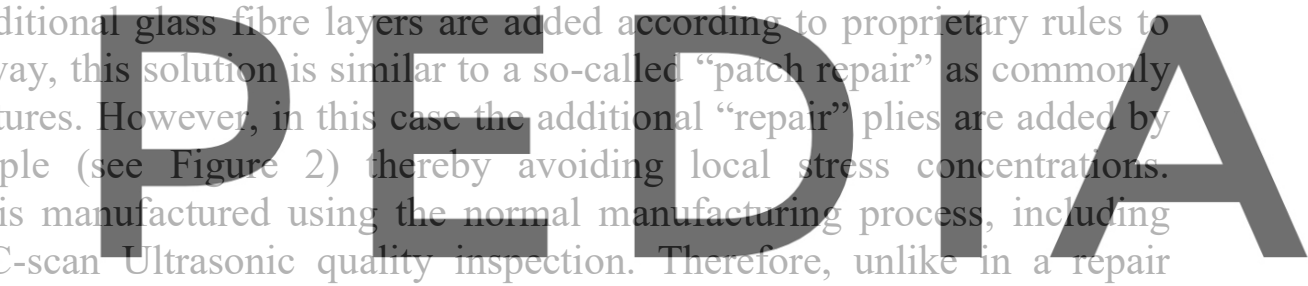
situation, the material quality is identical to that of the undisturbed laminate. Further details

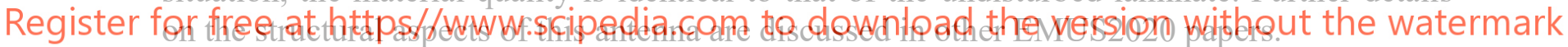

\section{DESIGN CHALLENGES}

The design of the integrated VHF slot antenna provokes various design challenges, most of which being trade-offs in structural and EM antenna performances. One of the main design criteria is the overall weight of the antenna, including structural reinforcements and PPR. This introduces several trade-offs, for instance when minimizing the thickness (i.e. weight) of the PPR. Reduction of the thickness comes at the cost of the antenna performance, e.g. for a thinner antenna it becomes harder to obtain low $S_{11}$ results over a large bandwidth. Another trade-off that is largely dominated by the weight and overall dimensions of the antenna, is the choice of material for the PPR interior. An air-filled PPR seemed to yield better $S_{11}$ results in simulations. Additionally, its weight will be minimal, however the required size of the slot is higher, giving rise to extra weight in terms of structural reinforcements. Moreover, for a larger PPR it will be more challenging to fit between the frames and stringers of an aircraft. Within the ACASIAS project the choice was made to create two versions of a dielectric filled PPR, a thick and thin one, to demonstrate corresponding weight vs. antenna performance trade-offs.

The feeding structure also introduces tradeoffs. Ideally, the feed is integrated into the PPR. 
However, this would make replacing the feed in case of a damage (due to for instance lightning) very hard. Therefore, in the demonstrator discussed in the next section a very thin FlexPCB feed was placed on the outside of the fuselage panel. Finally, also the use of a matching network introduces trade-offs. Such a network can improve the matching, but usually this comes at the cost of gain. However, in this case it is expected that the benefit for bandwidth is much more than the loss of gain.

\section{DEMONSTRATOR RESULTS}

Based on the theoretical designs obtained by simulations and several trade-off choices that were made in the ACASIAS consortium, a demonstrator integrated VHF antenna was built and measured. A parallel plate resonator was manufactured from PCB material. Two different PPRs were manufactured, named the 'thick PPR' and the 'thin PPR', the difference being the channel thickness. The overall thickness of the thick PPR is $0.07 \lambda_{0}$ (or $16.8 \mathrm{~cm}$ ), while for the thin PPR the thickness was $0.014 \lambda_{0}$ (or $35 \mathrm{~mm}$ ). Both PPRs were made up out of multiple PCB layers of $3.5 \mathrm{~mm}$ thickness. The substrate material was FR4, while the parallel plates were formed by copper ground planes on the PCB substrate. GKN Fokker Aerostructures manufactured an FML panel with a slot following the bookleafing principle introduced in section 3. Pictures of the FML panel with slot and the thin PPR are shown in Figure 3.

An Agilent E8386B VNA was used to measure the $S_{11}$ of the FML slot antenna including PPR. FlexPCBs manufactured by Trackwise were used to feed the antenna. Resulting $S_{11}$ for intended to optimize th equal to $13 \%(120-136$

illustrate that decreasin higher $S_{11}$ levels than f However, the red line indicates what can be

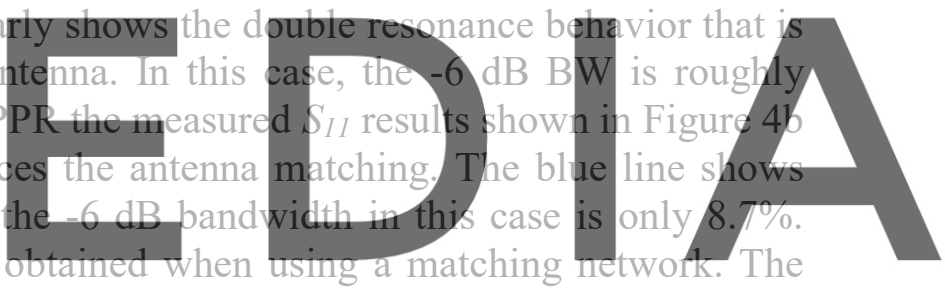
reflection coefficient is greatly improved, yielding a -6 dB BW of 22\% (120.9-148.5 MHz).

Register for free at https//www.scipedia.com to download the version without the watermark

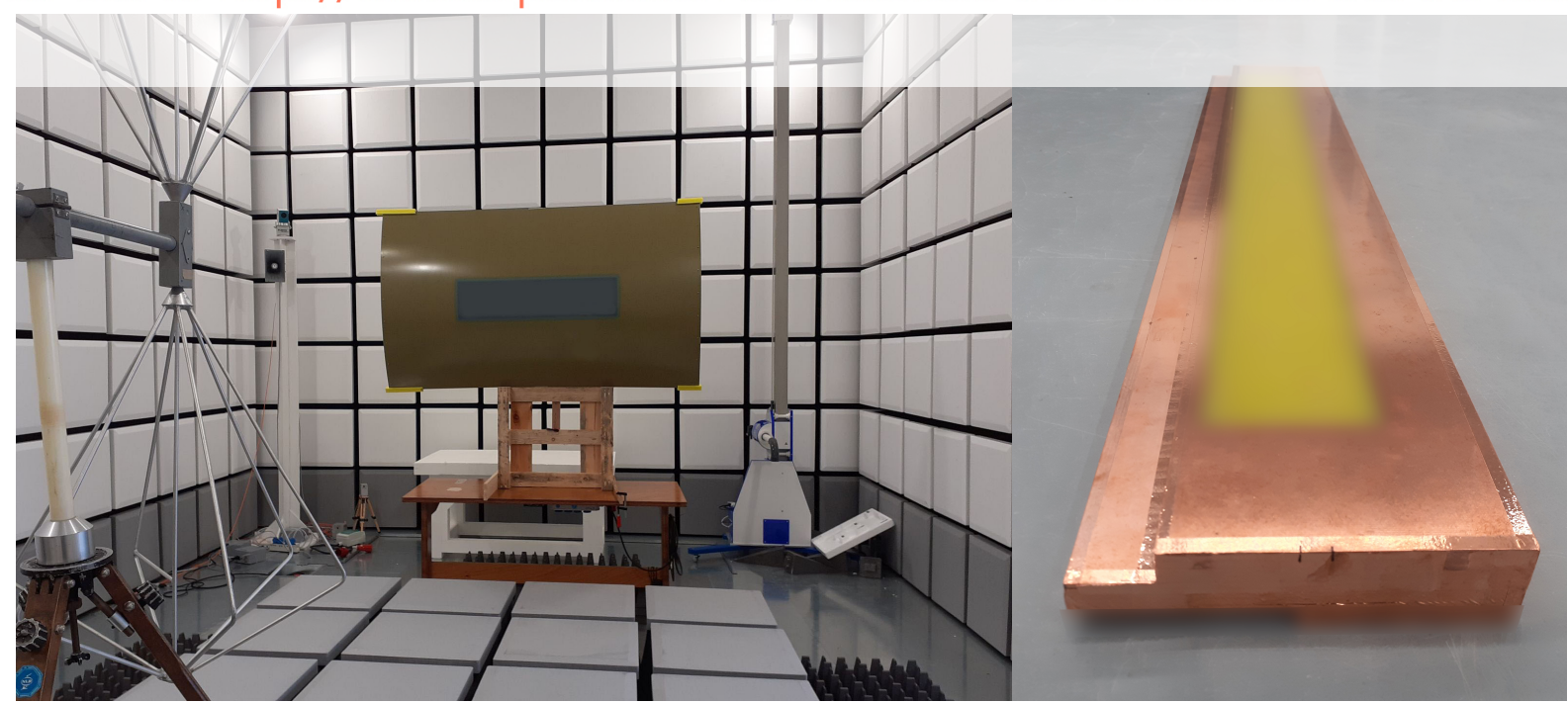

Figure 3: Pictures of (a) (left) the VHF antenna integrated into FML panel in radiation pattern test set-up, and (b) a close-up of the thin PPR manufactured from PCB material (details are blurred due to confidentiality). 

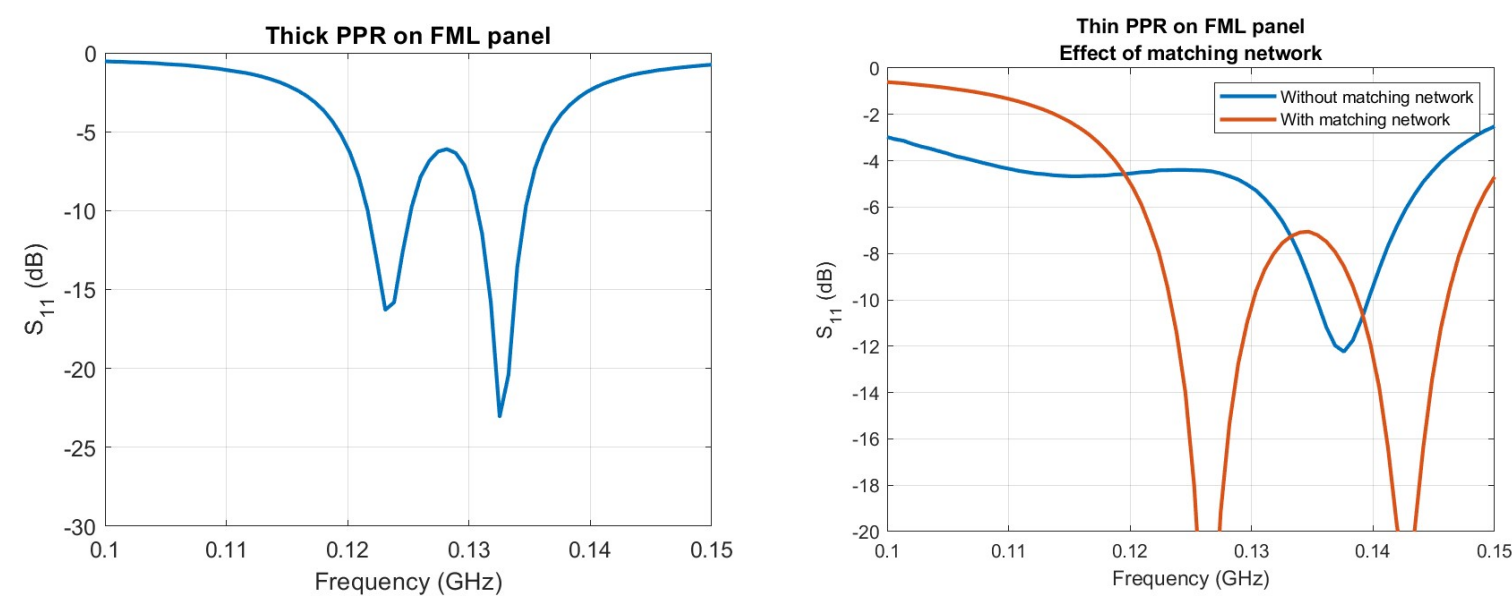

Figure 4: Measured $S_{11}$ for the slot integrated into an FML panel with (a) (left) a thick PPR, and (b) a thin PPR without (blue line) and with (red line) matching network.

\section{CONCLUSIONS}

This paper presents the design of an integrated VHF slot antenna with parallel plate resonator. The principles of EM design are explained, including slot antenna, microstrip feed and PPR. A bookleafing principle is applied to realize local reinforcements in the area of the slot antenna. This ensures that the structural quality of the FML panel with integrated antenna

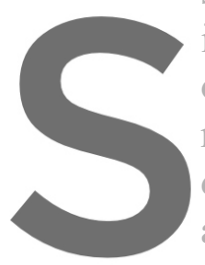
is not degraded. The ant of the most important manufactured, with whi
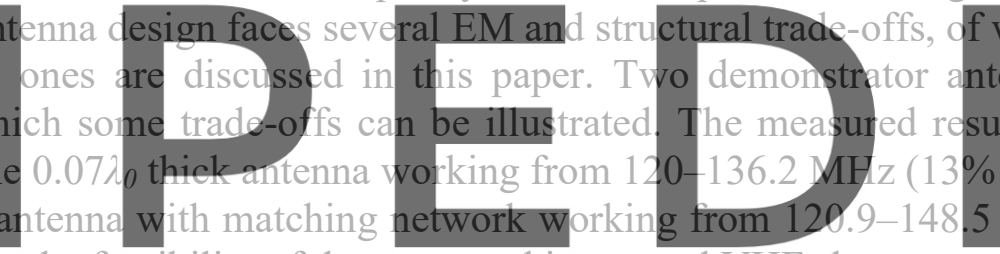
and one $0.014 \lambda_{0}$ thick a

$-6 \mathrm{~dB}$ BW). This proves the feasibility of the presented integrated VHF slot antenna.

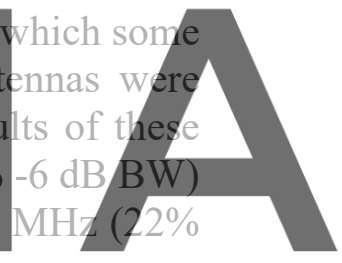

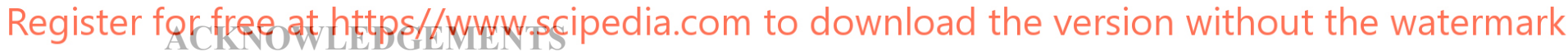

This project has received funding from the European Union's Horizon2020 research and innovation programme under grant agreement No.723167.

\section{REFERENCES}

[1] D. Sievenpiper, L. Zhang, R.F. Jimenez Broas, N.G. Alexópolous, E. Yablonovitch, "High-impedance electromagnetic surfaces with a forbidden frequency band", IEEE Trans. on Antennas and Prop. (1999), 47 (11): 2059-2074.

[2] J. Joubert, J.C. Vardaxoglou, W.G. Whittow and J.W. Odendaal, "CPW-fed cavity-backed slot radiator loaded with an AMC reflector", IEEE Trans. on Antennas and Prop. (2012), 60 (2): 735-742.

[3] H. Malekpoor and S. Jam, "Improved radiation performance of low profile printed slot antenna using wideband planar AMC surface", IEEE Trans. on Antennas and Prop. (2016), 64 (11): 4626-4638.

[4] J. Li, H. Shi and A. Zhang, "Unidirectional circularly polarized slot antenna backed with miniaturized AMC reflector", IEEE Int. Symp. on Antennas and Prop. \& USNC/URSI 
Nat. Radio Sc. Meeting, Boston, MA, USA, July 2018.

[5] W. Hong and K. Sarabandi, "Platform embedded slot antenna backed by shielded parallel plate resonator", IEEE Trans. on Antennas and Prop. (2010), 58 (9): 2850-2857.

[6] N. Behdad and K. Sarabandi, "A wide-band slot antenna design employing a fictitious short circuit concept", IEEE Trans. on Antennas and Prop. (2005), 53 (1): 475-482.
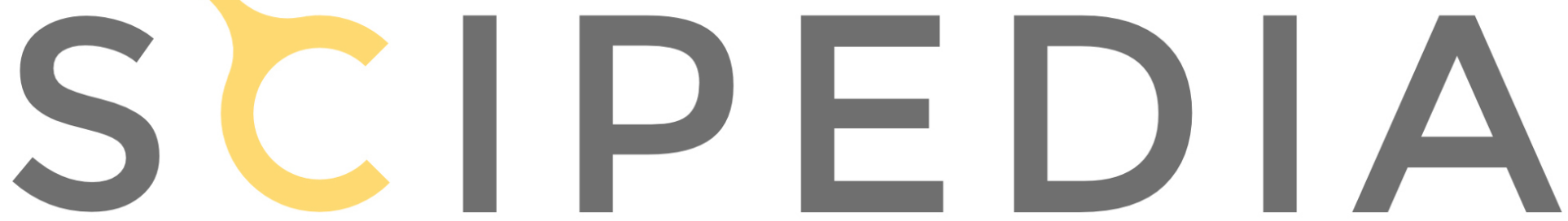

Register for free at https//www.scipedia.com to download the version without the watermark 